\title{
Structure of the Human Telomeric Stn1-Ten1 Capping Complex
}

\author{
Christopher Bryan ${ }^{1,2}$, Cory Rice ${ }^{1,3}$, Michael Harkisheimer ${ }^{1}$, David C. Schultz ${ }^{1}$, Emmanuel Skordalakes ${ }^{1,2,3 *}$ \\ 1 Gene Expression and Regulation Program, The Wistar Institute, Philadelphia, Pennsylvania, United States of America, 2 Department of Chemistry, University of \\ Pennsylvania, Philadelphia, Pennsylvania, United States of America, 3 Department of Biochemistry and Biophysics, University of Pennsylvania, Philadelphia, Pennsylvania, \\ United States of America
}

\begin{abstract}
The identification of the human homologue of the yeast CST in 2009 posed a new challenge in our understanding of the mechanism of telomere capping in higher eukaryotes. The high-resolution structure of the human Stn1-Ten1 (hStn1-Ten1) complex presented here reveals that hStn 1 consists of an OB domain and tandem C-terminal wHTH motifs, while hTen 1 consists of a single $\mathrm{OB}$ fold. Contacts between the $\mathrm{OB}$ domains facilitate formation of a complex that is strikingly similar to the replication protein A (RPA) and yeast Stn1-Ten1 (Ten1) complexes. The hStn1-Ten1 complex exhibits non-specific singlestranded DNA activity that is primarily dependent on $\mathrm{hStn} 1$. Cells expressing $\mathrm{hStn} 1$ mutants defective for dimerization with hTen1 display elongated telomeres and telomere defects associated with telomere uncapping, suggesting that the telomeric function of hCST is hTen1 dependent. Taken together the data presented here show that the structure of the hStn1-Ten1 subcomplex is conserved across species. Cell based assays indicate that hTen1 is critical for the telomeric function of hCST, both in telomere protection and downregulation of telomerase function.
\end{abstract}

Citation: Bryan C, Rice C, Harkisheimer M, Schultz DC, Skordalakes E (2013) Structure of the Human Telomeric Stn1-Ten1 Capping Complex. PLoS ONE 8(6): e66756. doi:10.1371/journal.pone.0066756

Editor: Arthur J. Lustig, Tulane University Health Sciences Center, United States of America

Received March 11, 2013; Accepted May 10, 2013; Published June 24, 2013

Copyright: (c) 2013 Bryan et al. This is an open-access article distributed under the terms of the Creative Commons Attribution License, which permits unrestricted use, distribution, and reproduction in any medium, provided the original author and source are credited.

Funding: The research was funded by the National Institutes of Health (NIH) National Institute of General Medical Sciences (NIGMS) (5 R01 GM088332-03) and The Wistar Cancer Center Support Grant (P30 CA10815). The funders had no role in study design, data collection and analysis, decision to publish, or preparation of the manuscript.

Competing Interests: The authors have declared that no competing interests exist.

*E-mail: skorda@wistar.org

\section{Introduction}

Telomeres are the guanosine rich DNA repeats at the ends of linear eukaryotic chromosomes that play a crucial role in protection and replication of the genome [1,2]. An assortment of telomere-associated proteins allows the cell to address the special challenges of telomere maintenance and replication $[3,4,5,6]$. In $S$. cerevisiae, the trimeric CST complex (sc CST, composed of Cdc13, $s c \mathrm{Stn} 1$ and $s c$ Ten 1 ) binds tightly and specifically to the telomeric G-overhang [7] and plays a critical role in telomere maintenance $[7,8,9,10]$. G-strand binding by scCST primarily depends on Cdc13, although telomeric DNA binding by $s c \mathrm{Stn} 1$ and $s c$ Ten 1 has also been proposed $[11,12,13]$.

Cdc 13 recruits telomerase to telomeres through binding to Est1, an interaction that is required for telomere extension in vivo $[14,15,16,17]$. However, the scCST complex negatively regulates telomere length by sequestering the telomeric overhang thus preventing access of telomerase to telomeres [9]. After G-strand extension, $s c \mathrm{CST}$ promotes telomeric C-strand fill-in by recruiting the DNA polymerase $\alpha$-primase to telomeres; a process mediated by the N-terminal domain of $\mathrm{Cdc} 13$ and the $\mathrm{C}$-terminal domain of Stn $1[10,18]$. In addition to its role in telomere replication, scCST caps telomeres to prevent recombination and exonucleolytic degradation events that could lead to genomic instability and checkpoint-dependent cell-cycle arrest in the G2/M phase $[7,19,20,21,22,23]$. Loss of any scCST protein component leads to telomere phenotypes associated with uncapped telomeres $[9,10,24]$.
Homologues of $s c \mathrm{Stnl}$ and $s c$ Tenl were identified in fission yeast by Martin V. et al. [25] in 2007 and have been subsequently discovered in a wide range of species, including plants and vertebrates including humans $[4,26]$. Like the $s c \mathrm{CST}$, the hCST (Ctcl, hStnl and hTenl) complex is predicted to contain multiple OB-folds and it binds telomeric overhangs with high affinity and some specificity $[4,27,28,29]$.

hStnl and hCtcl are also cofactors of DNA polymerase $\alpha$ primase that promote $\mathrm{C}$-strand synthesis at the telomeres [29,30,31. Loss of CST function in higher eukaryotes results in accumulation of excessive G-strand telomere DNA and the formation of extra-chromosomal t-circles, events associated with telomere uncapping $[4,27,32]$. Despite other similarities to the scCST complex, there is currently no evidence to suggest that hCST recruits telomerase to telomeres. Instead, hCST blocks telomerase access to the G-overhang by inhibiting its interaction with TPP1 [28], a component of the shelterin telomere maintenance complex [6]. Loss of any component of hCST results in telomerase-mediated telomere lengthening [28]. In contrast to $s c \mathrm{CST}$, some reports suggest a possible role for hCST in extra-telomeric DNA replication. hCST has been implicated in promoting genome-wide replication restart after fork stalling by promoting dormant replication origin firing [27,33]. Overall, hCST plays a role in telomere replication, and may also have a more general role under conditions of replicative stress. Despite the shared similarities between $s c \mathrm{CST}$ and hCST, the low sequence identity and reported functional differences between the two complexes raise questions regarding their structural and 
functional conservation in telomere length regulation and chromosome end protection.

To further elucidate the role of hCST at the telomeres, we solved the structures of hTenl in complex with the N-terminal domain of hStnl (hStnlN), and the C-terminal domain of hStnl $(\mathrm{hStn} 1 \mathrm{C})$ alone. These structures reveal that hStn 1-Ten l is a RPAlike complex and a structural homologue of Schizosaccharomyces pombe StnlTenl (spStnlTenl) and Candida tropicalis StnlTenl (ctStn1Ten1). DNA binding assays reveal that hStnl shows a robust ssDNA binding activity while hTen 1 binds ssDNA at a nonappreciable level $(\geq 15 \mu \mathrm{M})$. Functional assays of hStnl mutants defective for hTen 1 binding show telomere signal-free ends, longer and fragile telomeres, phenotypes associated with telomere length deregulation and a dysfunctional scCST complex. These data support a RPA-like DNA binding mechanism for hStn1-Tenl, and show that this complex plays a critical role in telomere maintenance.

\section{Results}

\section{Structure of the hStn1-Ten1 Complex}

To elucidate the function of the human telomeric Stn1-Tenl complex in telomere biology, we prepared the full-length hStnlTenl (Figure S1A), the truncated hStn $1 \mathrm{~N}-\mathrm{Ten} 1$ (Figure S1B) and hStnlC (Figure S1C) proteins to homogeneity. We subsequently solved the structures of the hStn1N-Tenl complex and hStnlC to $2.05 \AA$ and $1.65 \AA$ resolution respectively using mercury derivatives and the method of multi-wavelength anomalous dispersion (MAD) (Table 1 and 2). hStn $1 \mathrm{~N}$ adopts an OB fold, consisting of a $\beta$-barrel, composed of two three-stranded antiparallel $\beta$-sheets (with $\beta 3$ shared across both of the $\beta$-sheets), sandwiched by three $\alpha$-helices (Figure 1A). An indentation on the surface of the protein forms the putative substrate-binding pocket $(\mathrm{PBP})$ of the molecule. Helix $\alpha \mathrm{l}$ is located at the very $\mathrm{N}$-terminus of the domain and caps one end of the $\beta$-barrel. Helix $\alpha 2$, composed of approximately twelve non-conserved residues, is located between strands $\beta 5$ and $\beta 6$ and is partially visible in the electron density. A striking feature of $\mathrm{hStn} 1 \mathrm{~N}$ is a long $\alpha$-helix $(\alpha 3)$ located at the opposite end of the substrate-binding pocket of the OB-fold and runs parallel to the plane of the $\beta$-barrel (Figure 1A). hTen 1 adopts a similar OB fold consisting of five antiparallel $\beta$ strands folded into a $\beta$-barrel and flanked by two alpha helices. The first twelve $\mathrm{N}$-terminal residues comprise a long coil that sits atop the surface of the $\beta$-barrel (Figure 1B) and plays an important role in hStn1-Tenl assembly. Following the N-terminal coil is a short alpha helix $(\alpha 1)$ positioned at the edge of the interface of the two $\beta$-sheets that form the $\beta$-barrel providing stability to the $\mathrm{OB}$ fold. The $\beta$-barrel contains the classic puckered surface that usually comprises the substrate-binding pocket of $\mathrm{OB}$ fold proteins. Located at the opposite end of the $\beta$-barrels' puckered surface is a long C-terminal $\alpha$-helix ( $\alpha 2)$ that spans the entire length of the $\beta$-barrel, like $\alpha 3$ of hStn 1 (Figure 1A and $\mathbf{B}$ ). The G-terminal domain of hStnl consists of eleven $\alpha$-helices and four $\beta$-strands organized into two distinct winged helix-turn-helix (wHTH) motifs (Figure 1C) similar to $s c \mathrm{Stn} 1$ [34,35]. Surprisingly the RMSD between human and $s c$ StnlC (PDB ID: 3KEY and $3 \mathrm{~K} 10)$ is $6.1 \AA$ [36]. However, the inflated RMSD observed between the two structures arises in large part from the overall organization of the two wHTH motifs and not the fold of the protein. Structural alignment of the hStnlC and $s c \mathrm{Stn} 1 \mathrm{C}$, wHTH1 motifs shows that the hStnlC wHTH2 motif is rotated about $25^{\circ}$ away from the equivalent motif of $s c \mathrm{Stn} 1 \mathrm{C}$ (Figure S2B) suggesting structural flexibility between the two motifs especially in their substrate-free state. However, structural comparison of the equivalent hStnlC and $s c \mathrm{Stn} l \mathrm{C}$ (PDB ID: 3KEY or 3K10), wHTH motifs show RMSDs of $2.0 \AA$ and $3.3 \AA$ (DALI) for $\mathrm{wHTH}_{1}$ and $\mathrm{wHTH}_{2}$ respectively (Figure $\mathbf{S 2 C}$ and S2D), suggesting Stn1C structural conservation between the two organisms. The hStnl wHTH2 is most similar to the wHTH of human RPA32 (PDB ID: 1DPU) with an RMSD of $2.0 \AA$ (DALI)

(Figure S2E). This result was unexpected given that the ${ }^{\mathrm{HTH}} \mathrm{H}$ domain of $s c \mathrm{Stn} 1$ is most similar to the wHTH of RPA32 [34,35].

Full-length hStnl and hTenl form a stable heterodimer via the N-terminal portion of hStnl (Figure 2A). Contacts between the two proteins are mediated by extensive interactions between the C-terminal helices ( $\alpha 2$ and $\alpha 3$ of hTenl and hStnl respectively) and $\beta$-barrels of the two proteins (Figure $2 \mathbf{B}$ and $\mathbf{C}$ ). These two helices are highly conserved among spStn1-Tenl, ctStnl-Tenl hStn1-Ten1 and RPA14-RPA32 where they are also involved in protein oligomerization [34,37]. Many of the conserved residues making contacts between hStn $1 \mathrm{~N}$ and hTenl are located on the C-terminal helices and the $\beta$-barrels of these proteins. In particular, residues V159, W160, I164, M167 and L168 of helix $\alpha 3$ and flanking coils of hStnl form an extensive hydrophobic patch that interacts with the conserved residues, M100, L104, L105 and I109, of $\alpha 2$ and flanking coils of hTen 1 (Figure 2D). Additional contacts between the $\alpha$-helices of the two proteins are mediated by the conserved Y115 of $\alpha 2$ of hTenl. This residue is located at the interface of the two proteins and makes extensive hydrophobic interactions with the side chains of Y49, P171 and Y174 of hStnlN (Figure 2D). Additional contacts between the two proteins involve the surface of the $\beta$-barrels and the $\mathrm{N}$ terminal tail of hTen1, which runs along the interface of the two domains and makes extensive interactions with both proteins (Figure 2G). In particular, R27 ( $\beta 1)$ and R119 ( $\alpha 2)$ of hTen 1 form salt bridges with D78 $(\beta 2)$ and D33 $(\alpha 1)$ of hStnl, respectively (Figure 2E). The conserved residue M167 of hStnl extends toward the interface between $\alpha 2$ and the $\beta$-barrel of hTen 1 and makes extensive contacts with Y9 of the N-terminal coil and L105, A108 and I109 of $\alpha 2$ (Figure 2D). Interestingly, hStnl-Tenl domain organization positions the substrate-binding pockets of each subunit on the same side of the heterodimer, creating an extensive substrate-binding pocket (Figure 2G).

A search in the PDB database using the Dali server shows that the structure of the hStn1N-Tenl complex is strikingly similar to the $S$. pombe and C. tropicalis Stn1-Tenl telomeric complexes with an RMSD of $2.1 \AA$ and $2.9 \AA$ respectively (Figure S3A and B), indicating significant structural conservation of these telomeric complexes between humans and $s p$ StnlTenl and $c t$ Stn 1 Ten 1. There is also a degree of structural conservation between hStn $1 \mathrm{~N}$ Tenl and the human RPA32-RPA14 complex (PDB ID:1QUQ) with an RMSD of $3.1 \AA$ (Figure S3G).

\section{hStn1-Ten1 DNA Binding Properties}

Given that hCST binds single stranded DNA and hStnl and hTenl adopt OB folds, we sought to determine whether one or both proteins contribute to the ssDNA binding activity of the hCST complex. Using fluorescence polarization assays, we tested whether full-length hStnl, hTenl, and the hStnl-Tenl complex bound to telomeric and non-telomeric DNA oligomers of various lengths. hStnl binds ssDNA substrates 18 bases or longer (telomeric (tel18) and non-telomeric (rand18) - Table 3) with an affinity of $\sim 0.2 \mu \mathrm{M}$ (Figure 3A) while its affinity for probes shorter than 3 telomeric repeats was significantly decreased. For example it binds 2 telomeric repeats (tell2- Table 3) with $\sim 1 \mu \mathrm{M}$ and its binding affinity for a $8 \mathrm{mer}$ (tel8- Table 3) was too weak to determine (Figure 3D). 
Table 1. hStn1N-Ten1 Complex Data Collection and Refinement statistics.

\begin{tabular}{|c|c|c|c|}
\hline \multirow[t]{2}{*}{ Data collection } & \multirow[t]{2}{*}{ Native } & \multicolumn{2}{|c|}{ Hg Derivative (MAD Phasing) } \\
\hline & & E1 & E2 \\
\hline Wavelength $(\AA)$ & 1.1 & 1.0076 & 1.1 \\
\hline Space group & $\mathrm{P} 2,2,2$ & $\mathrm{P} 2{ }_{1} 2,2$ & $\mathrm{P} 2,2_{1} 2$ \\
\hline \multicolumn{4}{|l|}{ Cell dimensions } \\
\hline$a, b, c(\AA)$ & 130.658 .187 .6 & 129.855 .989 .0 & $130.0,56.189 .0$ \\
\hline Resolution (Å) & $20-2.05(2.16-2.05)$ & $50-2.15(2.19-2.15)$ & $50-2.15(2.19-2.15)$ \\
\hline$R_{\text {sym }}$ & $7.5(43.5)$ & $6.3(43.0)$ & $6.6(49.6)$ \\
\hline$|/ \sigma|$ & $9.7(1.6)$ & $19.5(1.8)$ & $17.7(2.1)$ \\
\hline Completeness (\%) & $100(100)$ & $99.9(99.9)$ & $99.8(99.6)$ \\
\hline Redundancy & $5.8(5.9)$ & $3.4(3.4)$ & $3.4(3.1)$ \\
\hline \multicolumn{4}{|l|}{ Phasing Analysis } \\
\hline Resolution (Å) & & & $50-2.7$ \\
\hline Mean figure of merit (FOM) & & & 0.49 \\
\hline Score (solve) & & & 28.8 \\
\hline Number of sites & & & 8 \\
\hline \multicolumn{4}{|l|}{ Refinement } \\
\hline Resolution (Å) & $20-2.05$ & & \\
\hline No. reflections & 40467 & & \\
\hline$R_{\text {work } /} R_{\text {free }}$ & $20.9 / 25.2$ & & \\
\hline \multicolumn{4}{|l|}{ No. atoms } \\
\hline Protein & 4215 & & \\
\hline Water & 190 & & \\
\hline \multicolumn{4}{|l|}{ B-factors } \\
\hline Protein & 37 & & \\
\hline Water & 40 & & \\
\hline \multicolumn{4}{|l|}{ R.m.s deviations } \\
\hline Bond lengths $(\AA)$ & 0.009 & & \\
\hline Bond angles $\left({ }^{\circ}\right)$ & 1.445 & & \\
\hline \multicolumn{4}{|l|}{ Ramachandran plot (\%) (Coot) } \\
\hline Preferred & 93.68 & & \\
\hline Allowed & 5.14 & & \\
\hline
\end{tabular}

hTenl also contains an OB fold with a similar structure to hStnl, but interacts with ssDNA much more weakly, as FP showed only a slight shift at protein concentrations of at least $15 \mu \mathrm{M}$ or higher (Figure 3B and D). The ssDNA binding activity of hTenl could not be detected using a tel30 probe, but was visible for a tell 18 probe and best for a tell 2 probe (Figure 3B and Table 3). This is likely due to the large dynamic range of small probes in FP experiments. The weak ssDNA binding activity of hTenl is supported by structural conservation analysis, as the residues that form the putative DNA binding pocket (PBP) of hTenl are poorly conserved (Figure 4B). This suggests that hTenl is not subject to evolutionary pressure to maintain a strong ssDNA binding activity. In fact, the only conserved surface area of hTenl is involved in hStnl binding (Figure 4C). In contrast, hStnl's putative ssDNA binding pocket is composed of several highly conserved, solvent accessible residues (Figure 4E).

Interestingly, the hStnl-Tenl complex exhibited non-specific ssDNA binding activity but with an affinity lower than that observed for hStnl alone. For probes of at least 18 nucleotides or longer the binding affinity of hStnl-Tenl is $\sim 2 \mu \mathrm{M}$, while we could not determine a $\mathrm{K}_{\mathrm{d}}$ for the tell 2 probe (Figure $4 \mathrm{C}$ and $\mathbf{D}$ and Table 3). The difference (10 fold) in DNA binding affinity for the tell8 between hStnl alone and the hStnl-tenl complex can be explained as follows; the minimum DNA length required for maximum hStnl-DNA binding $(\sim 200 \mathrm{nM})$ is 18 bases, which is most likely sufficient to bind more than one hStnl molecule. Binding of multiple hStnl molecules to a single probe enhances the FP signal, leading to a lower calculated $\mathrm{Kd}$. It is worth noting that the affinity of hStnl for the tel12 probe (which most likely binds one hStnl molecule) is comparable to the affinity of hStnlTenl for tel18. It is therefore likely that a single hStnl molecule has similar binding affinity to the hStnl-Tenl complex.

\section{Mutants that Disrupt hStn1-Ten1 Dimerization in vitro}

To determine the role of hStnl-Tenl complex assembly in telomere function we designed single and double mutants of conserved residues (Figure S4A and $B$ ) required for hStn 1-Ten 1 heterodimerization. We carried out isothermal titration calorim- 
Table 2. hStn $1 \mathrm{C}$ data collection, phasing and refinement statistics.

\begin{tabular}{|c|c|c|c|}
\hline \multirow[t]{2}{*}{ Data Collection } & \multirow[t]{2}{*}{ Native } & \multicolumn{2}{|c|}{ Hg Derivative (MAD Phasing) } \\
\hline & & E1 & E2 \\
\hline Wavelength $(\AA)$ & 1.1 & 1.0076 & 1.1 \\
\hline Space group & $\mathrm{P} 22_{1} 2_{1} 2_{1}$ & $\mathrm{P} 22_{1} 2_{1} 2_{1}$ & $\mathrm{P} 2{ }_{1}{ }_{1} 2_{1}$ \\
\hline \multicolumn{4}{|l|}{ Cell dimensions } \\
\hline$a, b, c(\AA)$ & 28.876 .6114 .4 & 28.876 .5113 .9 & 28.876 .7114 .1 \\
\hline Resolution ( $(\AA)$ & $20-1.6(1.69-1.6)$ & $50-1.6(1.69-1.6)$ & $50-1.6(1.69-1.6)$ \\
\hline$R_{\text {sym }}$ & $5.9(48.6)$ & $6.4(50.2)$ & $5.5(29.9)$ \\
\hline $\mid / \sigma l$ & $13.8(1.5)$ & $13.6(1.5)$ & $14.9(2.4)$ \\
\hline Completeness (\%) & $99.9(99.8)$ & $96.4(91.3)$ & $95.1(80.4)$ \\
\hline Redundancy & $5.6(4.0)$ & $3.0(2.9)$ & $2.9(2.5)$ \\
\hline \multicolumn{4}{|l|}{ Phasing Analysis } \\
\hline Resolution $(\AA)$ & & & $50-2.5$ \\
\hline $\begin{array}{l}\text { Mean figure of merit } \\
\text { (FOM) }\end{array}$ & & & 0.42 \\
\hline Score (solve) & & & 30.5 \\
\hline Number of sites & & & 2 \\
\hline \multicolumn{4}{|l|}{ Refinement } \\
\hline Resolution ( $(\AA)$ & $20-1.6$ & & \\
\hline No. reflections & 32625 & & \\
\hline$R_{\text {work } /} R_{\text {free }}$ & $20.7 / 21.6$ & & \\
\hline \multicolumn{4}{|l|}{ No. atoms } \\
\hline Protein & 1350 & & \\
\hline Water & 98 & & \\
\hline \multicolumn{4}{|l|}{ B-factors } \\
\hline Protein & 17.9 & & \\
\hline Water & 21.0 & & \\
\hline \multicolumn{4}{|l|}{ R.m.s deviations } \\
\hline Bond lengths ( $(\AA)$ & 0.008 & & \\
\hline Bond angles $\left({ }^{\circ}\right.$ ) & 1.047 & & \\
\hline \multicolumn{4}{|l|}{$\begin{array}{l}\text { Ramachandran plot } \\
\text { (\%) (Coot) }\end{array}$} \\
\hline Preferred & 97.56 & & \\
\hline Allowed & 2.44 & & \\
\hline
\end{tabular}

etry (ITC) experiments using pure, homogeneous, wild type and mutant proteins of hStnl and Tenl (Figure S5) in order to gauge the effect of these mutants on hStnl-Tenl hetrodimerization. The wild type hStnl-Tenl complex assembles with a dissociation constant of $6.3 \mathrm{nM}($ Figure $\mathbf{5 A})$. Single mutants showed moderate loss (2-5.5 fold) of binding affinity while double mutants abolished hStnlN-Tenl binding (Figure 5B - F). For example, the hTenl (R27Q) mutation, which disrupts a salt bridge with D78 of hStnl, (Figure 2E) leads to a 5.5 fold loss ( $33 \mathrm{nM}$ ) of affinity between the two proteins (Figure 5B and G). The hTenl (Y115A) mutation, which eliminates hydrophobic contacts with residues Y49, Y174 and P1 71 of hStn 1 (Figure 2D), leads to a 2.5 fold (14 nM) loss of binding affinity (Figure 5C). The hTenl (R119Q) mutation, which disrupts a salt bridge with D33 of hStnl (Figure 2D), leads to a 2-fold reduction in binding affinity $(13 \mathrm{nM})$ (Figure 5D). ITC experiments carried out using the double hStnl mutants, (D78A/ I164A) or (D78A/M167A) did not detect binding with wild-type
hTen 1 (Figures 5E and F). Both double hStn l mutants disrupt the highly conserved salt bridge formed between D78 and R27 of hStnl and hTenl respectively (Figure 2E). I164A disrupts contacts with the hydrophobic patch of hTenl consisting of residues M100, L104 and L105. M167A disrupts the extensive hydrophobic contacts involving Y9, L105, A108, and I109 of hTenl (Figures 2D).

\section{Disruption of the hStn1-Ten1 Dimer Results in Elongated Telomeres}

To investigate the role of hStnl-Tenl assembly in telomere maintenance, we introduced the $\mathrm{hStnl}(\mathrm{D} 78 \mathrm{~A} / \mathrm{M} 167 \mathrm{~A})$ and (D78A/M167A) double mutants that disrupt hStnl-Tenl heterodimerization, into HEK $293 \mathrm{~T}$ cells, and probed for telomere length defects using Southern blots. We knocked down endogenous hStnl expression in HEK 293T cells by lentiviral infection with anti-hStnl shRNA (shRNA-S2) (Figure S6). We prepared four hStnl knockdown cell lines by co-infecting with a $\mathrm{pLU}$ vector containing: a) no hStnl gene (hStnl-KD cell line), b) hStnl(WT) (hStnl-Rescue cell line) c) hStnl(D78A/I164A), and d) hStn1(D78A/M167A). We also created a mock-treated control cell line by co-infecting HEK 293T cells with a shRNA targeting green fluorescent protein (GFP), and the pLU vector that does not carry any gene. We also prepared a cell line expressing anti-hTen 1 shRNA (shRNA-T1) and the pLU vector without any gene (hTenl-KD) in order to determine the effect of simple hTenl knockdown (Figure S6). The ectopic hStnl and hTenl genes included silent mutations conferring resistance to the above shRNAs. None of the cell lines used in this study displayed any changes in growth rate or morphology after infection, and throughout the course of the experiment we applied antibiotic selection pressure to ensure that only successfully infected cells were able to propagate.

Southern blot analysis of DNA isolated from hTen 1-KD, hStn 1$\mathrm{KD}$ of various passages (6,9 and 12) showed a progressive increase in telomere length compared to mock-treated cells and hStn1Rescue (Figure 6A), in agreement with previous reports [4,28]. Surprisingly, the hStnl double mutants hStnl(D78A/I164A) and (D78A/M167A) show slightly longer telomeres than the hStn1KD (Figure 6B). The presence of longer telomeres in cells expressing hStnl(D78A/I164A) and (D78A/M167A), relative to knockdown alone, can be attributed to a dominant negative effect of the overexpressed hStnl mutants. The hStnl mutants were designed to specifically disrupt hTenl but not Ctcl binding. Saturation of hCtcl with mutant hStnl suppresses the activity of trace amounts of endogenous hStnl produced under the shRNA knockdown, leading to a more severe phenotype.

\section{Disruption of the hStn1-Ten1 Complex Results in Chromosomal Abnormalities Associated with Dysfunctional Telomeres in vivo}

Previous reports have shown that cells with defective hCST due to either hStnl or hCtcl knockdown show elevated levels of telomere signal-free ends [27] as well as fragile telomeres [33]. To further establish the role of the hStn1-Ten 1 assembly in telomere maintenance, we asked if the $\mathrm{hStn} 1$, dimerization double mutants (D78A/I164A) and (D78A/M167A) exhibit a similar phenotype to simple knockdowns using fluorescence in situ hybridization (FISH) analysis. We prepared metaphase spreads of these cell lines by fixing the chromosomes to microscope slides with formaldehyde, and hybridizing telomeres with a Cy5-labelled peptide nucleic acid (PNA) probe targeting human telomeric repeats (PNA Bio, Inc.). Chromosomal DNA was stained with DAPI and photographs 
A

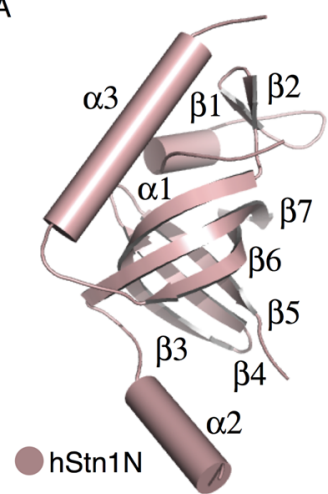

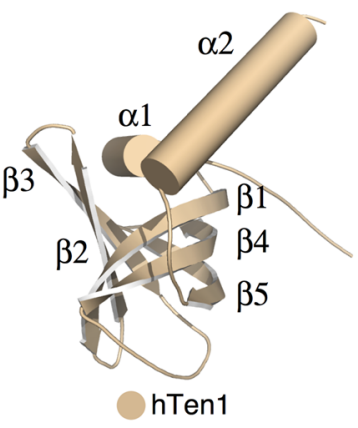

C

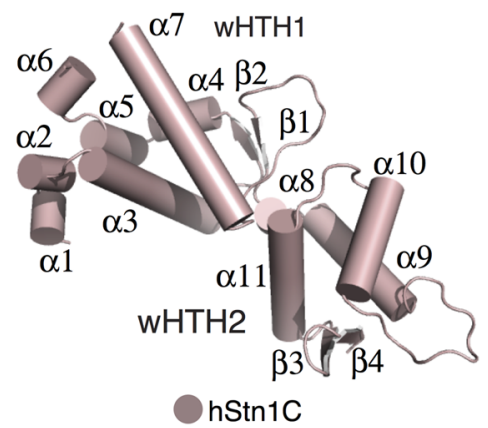

Figure 1. Crystal structures of hStn 1 and hTen1. (A) The N-terminal domain of hStn1, (B) full-length hTen1, and (C) The C-terminal domain of hStn1.

doi:10.1371/journal.pone.0066756.g001

were taken using the $100 \times$ objective of a Nikon E600 upright fluorescent microscope.

We counted the frequency of telomere signal-free ends, chromosome fusions, and fragile telomeres three weeks (passage 8) after infection. Knockdown of hStnl or hTenl led to elevated levels of chromosomes with missing telomeres (Figure 7A, B and C). This phenotype was rescued by reintroducing $\mathrm{hStn} l(\mathrm{WT})$, but not the hStnl mutants (D78A/I164A and D78A/M167A) defective of hTenl binding. Mock-treated (wild type) cells showed
$6.2 \%$ of chromosomes with missing telomeres, compared with $21.9 \%$ in hStnl-KD, and $24.0 \%$ in hTen 1 knockdown (Figure 7D). Reintroduction of hStnl(WT) to hStnl-KD cells reduced the number of missing chromosomes to $7.4 \%$, while overexpression of the hStn1(D78A/I164A) or (D78A/M167A) double mutants led to a significant increase in the number of missing telomeres $(24.6 \%$ and $19.5 \%$ respectively) (Figure 7D). We also found elevated levels of fragile telomeres in cells overexpressing hStnl mutants defective for hTenl dimerization

A

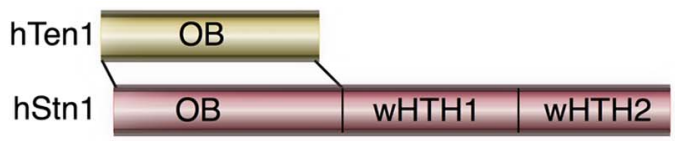

B

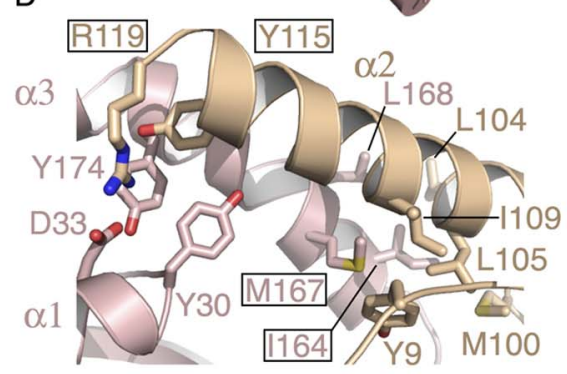

C

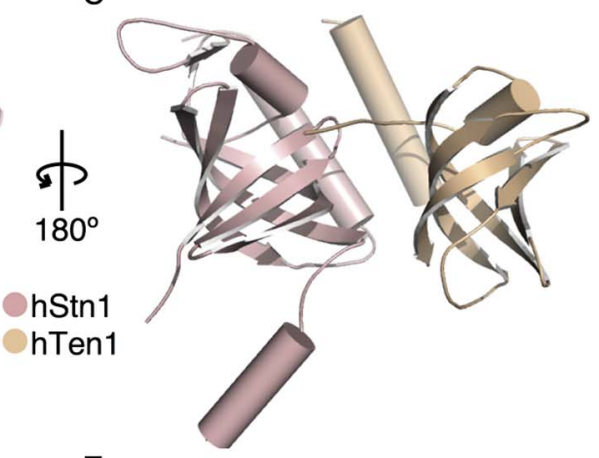

E

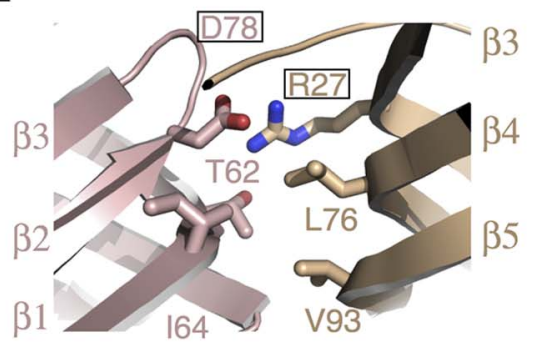

Figure 2. Structure of the hStn $1 \mathbf{N}$-Ten 1 heterodimer. (A) Primary structures of hStn1 and hTen 1 showing interacting domains. (B) Crystal structure of the hStn1-Ten1 dimer in cartoon representation looking down the interface of the two subunits. (C) View of the hStn1-Ten1 dimer rotated $180^{\circ}$ to highlight the arrangement of OB fold putative DNA binding pockets. (D) Dimerization contacts between the hStn $1 \mathrm{~N}$ and full-length hTen 1 C-terminal helices $\alpha 3$ and $\alpha 2$ respectively. Residues mutated in this study for ITC and cell based assays are shown in boxes. (E) Dimerization contacts between the $\beta$-barrels of hStn1N and hTen1.

doi:10.1371/journal.pone.0066756.g002 
Table 3. DNA oligos used in this study.

\begin{tabular}{ll}
\hline Oligo & Sequence \\
\hline Tel30 & TAGGGTAGGGTAGGGTTAGGGTTAGGG \\
Tel18 & TAGGGTAGGGTAGGG \\
Rand18 & GTTACGAAATACGGACAC \\
Tel12 & TAGGGTAGGG \\
Tel8 & TAGGGT \\
\hline doi:10.1371/journal.pone.0066756.t003
\end{tabular}

(Figure 7A, B and C). However, the levels of fragile telomeres in hStnl and hTenl knockdown cells were not significantly elevated over mock treated cells or hStnl-Rescue (Figure 7E). The increased number of TFE chromosomes observed for the hStn 1 dimerization mutants (D78A/I164A) and (D78A/M167A) compared to hStnl and hTen 1 knockdown cell lines can most likely be explained by a dominant negative phenotype of the overexpressed

A
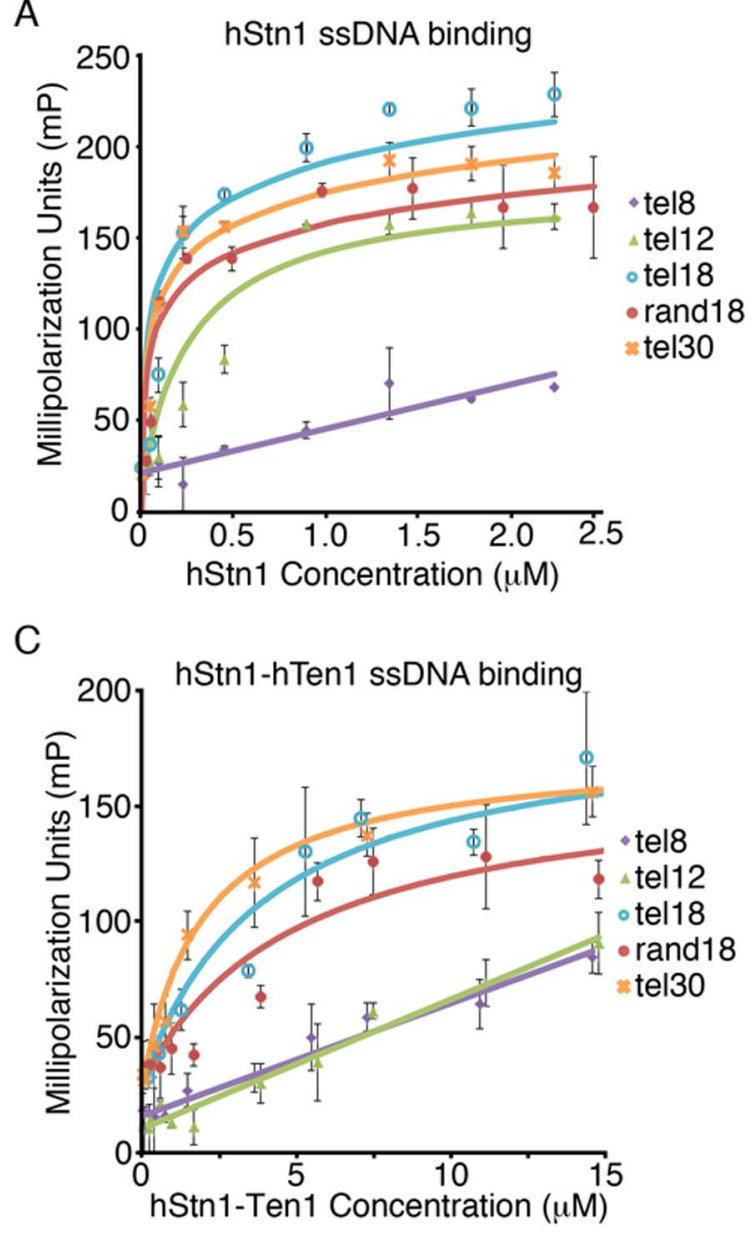

mutant as discussed earlier. We did not observe a significant change in the rate of telomere fusions across any of our cell lines.

\section{Discussion}

The identification of a human homologue of yeast CST in 2009 posed an interesting challenge for the telomere field as we need to rethink existing telomere capping models in vertebrates [4]. The results presented here provide evidence supporting structural and functional conservation of the CST complex across distant species, and provide insight into the role of the hStn1-Tenl complex at telomeres. Structural overlays of hStnl-Tenl with spStnl-Tenl (PDB ID 3KF6) and $c t$ Stn1-Tenl (PDB ID 3KF8) show a surprisingly high degree of similarity between these complexes, given the evolutionary distance and low sequence identity between these species. We observed that the hStnl-Tenl complex has striking structural similarities to both $s p$ Stn1-Tenl and $c t$ Stn 1Ten1. This close structural relationship indicates that they likely carry out a conserved function at telomeres.

The fact that Stnl-Tenl and RPA are structurally similar and share some functional aspects of DNA processing [30,38,39,40] raises the question as to why cells need both complexes. Current
B

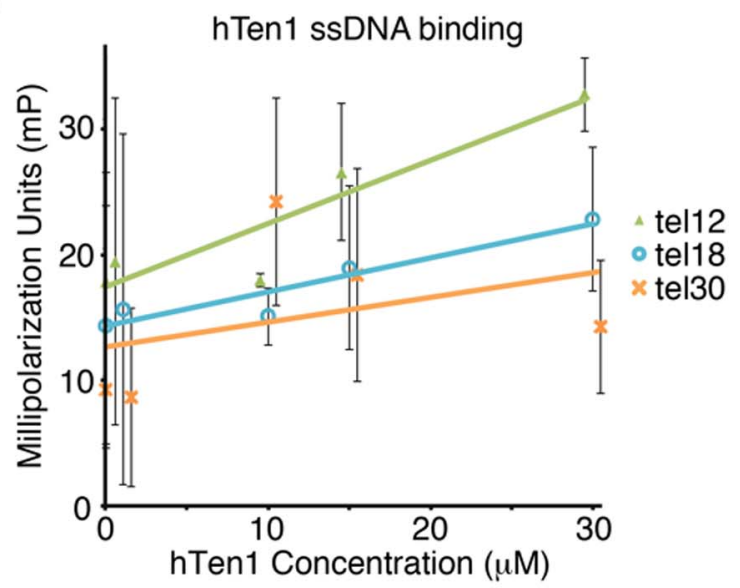

D

\begin{tabular}{|c|c|c|}
\hline \multicolumn{3}{|c|}{ hStn1, hTen1, and hStn1-Ten1 DNA Binding } \\
\hline Protein & DNA Substrate & $\mathrm{Kd}(\mu \mathrm{M})$ \\
\hline \multirow[t]{5}{*}{ hStn1 } & Tel30 & 0.2 \\
\hline & Tel18 & 0.2 \\
\hline & Rand18 & 0.3 \\
\hline & Tel12 & 2 \\
\hline & Tel8 & $\mathrm{WB}^{*}$ \\
\hline \multirow[t]{3}{*}{ hTen1 } & Tel30 & WB \\
\hline & Tel18 & WB \\
\hline & Tel12 & WB \\
\hline \multirow[t]{5}{*}{ hStn1-Ten1 } & Tel30 & 2 \\
\hline & Tel18 & 2 \\
\hline & Rand18 & 3 \\
\hline & Tel12 & WB \\
\hline & Tel8 & WB \\
\hline$B=$ & ing, no K & \\
\hline
\end{tabular}

\section{Bryan_Figure 3}

Figure 3. DNA binding properties of hStn1, hTen 1 and the hStn1-Ten 1 complex. Fluorescence Polarization (FP) data for (A) hStn1 (B) hTen1 and (C) the full length hStn1-Ten1 complex with ssDNA of various lengths (tel8, tel12, tel18, rand18, tel30- Table 3) (D) Table of hStn1, hTen1 and full-length hStn1-Ten1, ssDNA dissociation constants $\left(\mathrm{K}_{\mathrm{d}}\right)$ calculated from the FP data of panels $\mathrm{A}, \mathrm{B}$ and $\mathrm{C}$. doi:10.1371/journal.pone.0066756.g003 
A

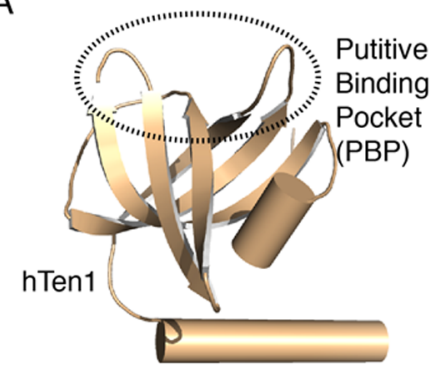

D

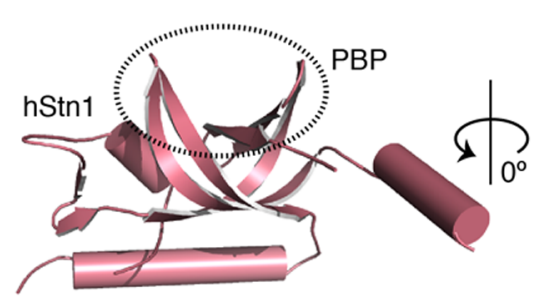

B

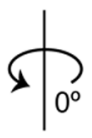

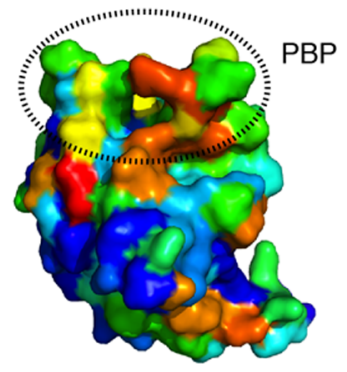

C

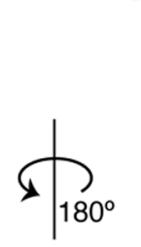

hStn1-interacting surface

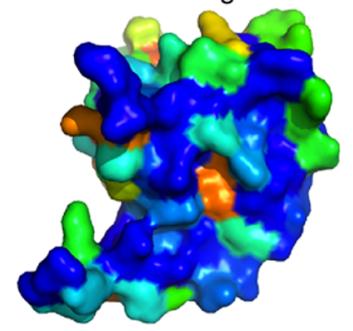

$\mathrm{F}$

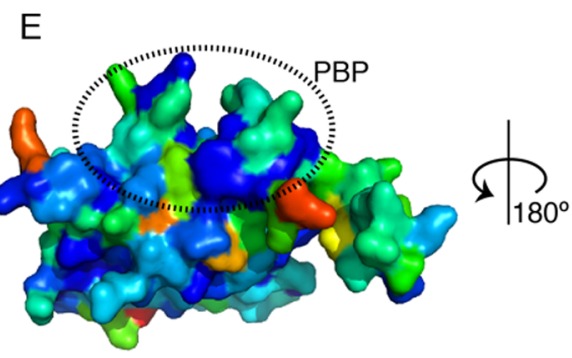

hTen1-interacting surface

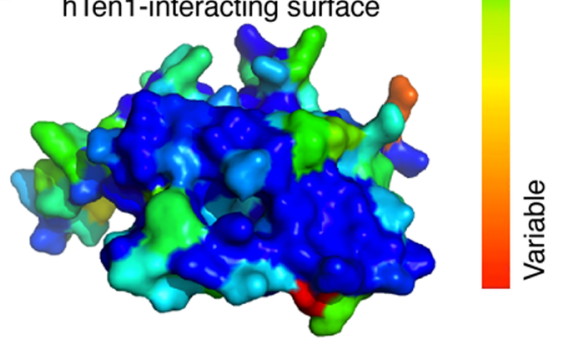

Bryan_Figure 4

Figure 4. hStn 1 and hTen 1 surface aminoacid conservation. (A) Tertiary structure (cartoon) of hTen1 highlighting the putative DNA binding pocket (PBP) with a dashed circle. (B and C) Conservation map for the PBP and the hStn1-interacting surface of hTen1 respectively. Blue indicates residue conservation and red, residues that are variable. (D) Tertiary structure (cartoon) of hStn1 highlighting the PBP with a dashed circle. (E and F) Conservation map for the putative binding pocket of hStn1 and the hTen1-interacting surface respectively. doi:10.1371/journal.pone.0066756.g004

evidence suggests that CST acts as a telomere specific version of the RPA complex, promoting efficient replication of telomeres [11]. hCST binds preferentially to the mammalian telomeric Gstrand [28], and has been shown to localize to $\sim 20 \%$ of telomeres [4]. Telomeric DNA poses a challenge to the normal replication machinery due to its repetitive sequence and the formation of Gquadrupex structures upon unwinding duplex DNA [41,42,43]. It may be the case that RPA is unable to resolve these structures and assist in the pol $\alpha$ - dependent DNA replication, so a specialized protein is required to promote efficient telomere replication. In support of this idea, disruption of CST has been shown to lead to delayed telomere replication and formation of telomere doublets, which may indicate replication fork stalling and collapse in the telomeric region [27]. CST may have an additional function in protecting telomere ends from DNA damage response by excluding RPA from binding to telomeric DNA, as RPA is capable of activating ATR-mediated DNA damage response, an activity that is undesirable at telomeres [44]. This notion is supported by the fact that in $S$. cerevisiae and humans CST association with telomeres reaches a maximum in the late $\mathrm{S}$ to early G2/M phase when G-overhangs are longest $[28,45,46]$.

Structural data reveal subtle yet important differences between hStn1-Tenl and Rpa32-Rpa14 that may provide an explanation to the above ideas. The most glaring difference between hStn 1 and RPA32 is that hStnl has 2 wHTH motifs C-terminal to the OB fold, while RPA32 only has one. wHTH2 of hStn1 is structurally similar to the wHTH of RPA32, so the presence of ${ }_{w} H T H 1$ may partially account for differential function between RPA and CST. In both RPA and Stn1, the OB fold is a ssDNA-binding domain while the C-terminal domain is important for protein-protein interactions. The additional wHTH motif in hStnl may allow interaction with a different set of proteins that function at telomeres such as Ctcl. Functional differences between hCST and RPA could also be attributed to lack of sequence identity and size of the RPA70 (616 aminoacids) and hCtcl (1217 aminoacids), the large subunits of each complex respectively. There is little mechanistic and no structural data on hCtcl, so further studies are necessary to determine the role that it is playing in the function of the complex.

The data presented here shows that cell lines defective of hStn 1Tenl heterodimerization exhibit telomere dysfunction in the form of elongated telomeres (Figure 6), telomere signal-free ends, and fragile telomeres (Figure 7), phenotypes associated with defective hCST [4,27]. This reinforces functional homology between hCST and $s c$ CST because both complexes are critical for telomere length regulation $[9,25,47]$. Our FP assays show that hStn 1 binds ssDNA with a $\mathrm{Kd}$ of $200 \mathrm{nM}$ alone and $2 \mu \mathrm{M}$ when in complex with hTenl. This data establishes a role for the hStn 1 protein in ssDNA binding, which facilitates telomere capping by hCST. In contrast, hTenl alone showed only a slight shift at protein concentrations of $\geq 15 \mu \mathrm{M}$ (Figure 3B). Although hTenl alone binds ssDNA extremely weakly, its localization to the telomeres by the CtclStnl complex would enhance hTenl - DNA binding by positioning the protein in proximity to the telomeric overhang. The structure of the hStnl-Tenl complex supports this notion as the organization of the hStnl-Tenl OB folds aligns the PBPs so ssDNA substrate could easily interact with both proteins (Figure 2G). However the weak DNA binding affinity of hTenl for ssDNA and the lack of amino acid conservation at its PBP raises important questions regarding the precise role of this protein in telomere maintenance. One possibility is that hTen 1 is indeed involved in ssDNA binding, which would suggest a conserved ssDNA binding mechanism with the scStnlTenl complex [11]. $s c \mathrm{Stnl}$ has been reported to bind ssDNA with a $2 \mu \mathrm{M}$ affinity, compared to $>6 \mu \mathrm{M}$ for $s c$ Tenl [12,13]. hCST-bound telomeric DNA must be accessible for C-strand synthesis by pol- $\alpha$, which requires hCST to hand-off ssDNA. The function of the homologous RPA complex has been proposed to depend on sequential binding and release of ssDNA by multiple $\mathrm{OB}$ domains [48]. The strongest-binding OB domains of RPA are at the 5' end 
A

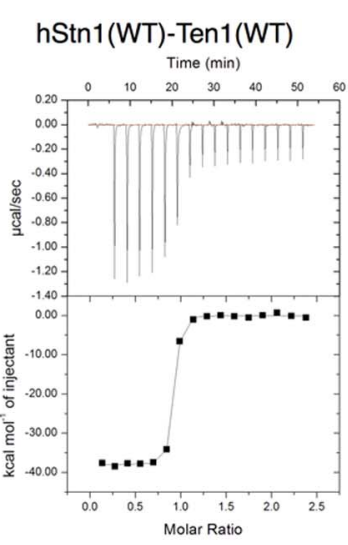

D

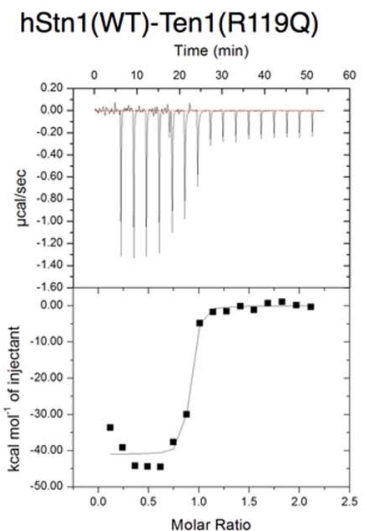

B

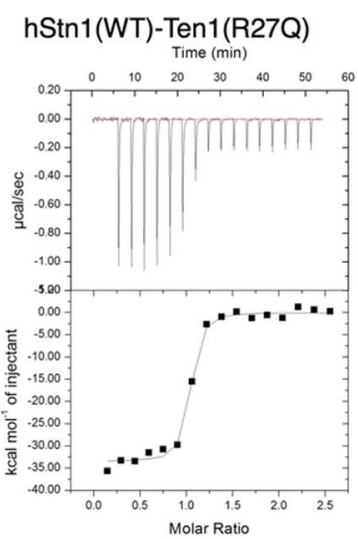

E

hStn1(D78A/1164A)-Ten1(WT)

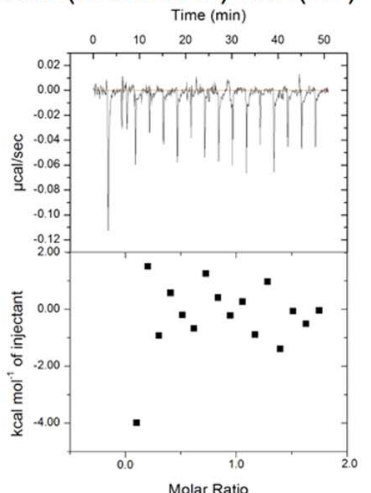

C

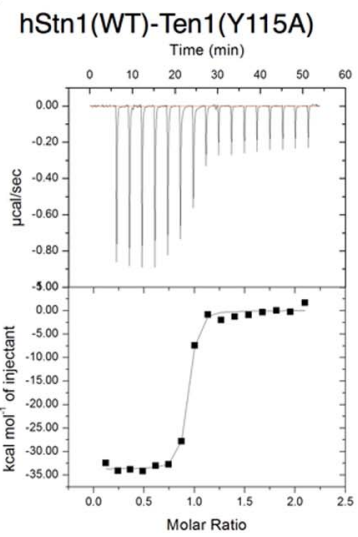

F

hStn1(D78/M167)-Ten1(WT)

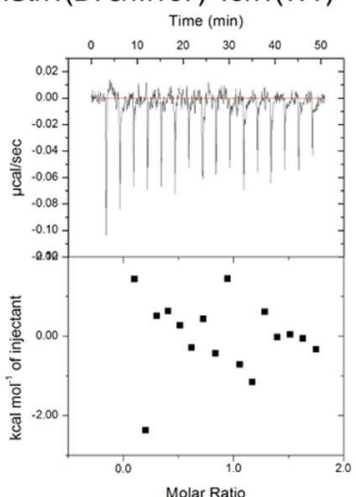

G

\begin{tabular}{|c|c|c|}
\hline $\begin{array}{c}\text { Stn1 } \\
\text { Construct }\end{array}$ & $\begin{array}{c}\text { Ten1 } \\
\text { Construct }\end{array}$ & $\begin{array}{c}\mathrm{Kd} \\
(\mathrm{nM})\end{array}$ \\
\hline $\mathrm{WT}$ & $\mathrm{WT}$ & 6.3 \\
\hline $\mathrm{WT}$ & $\mathrm{R} 27 \mathrm{Q}$ & 33 \\
\hline $\mathrm{WT}$ & $\mathrm{Y} 115 \mathrm{~A}$ & 14 \\
\hline $\mathrm{WT}$ & $\mathrm{R} 119 \mathrm{Q}$ & 13 \\
\hline D78A/164A & $\mathrm{WT}$ & $\mathrm{ND}^{\star}$ \\
\hline D78A/M167A & $\mathrm{WT}$ & $\mathrm{ND}^{*}$ \\
\hline *ND - No Binding detected \\
\hline
\end{tabular}

Figure 5. Isothermal titration calorimetry (ITC) data of hStn 1 and hTen 1 association. (A) hStn1(WT) with hTen1(WT). (B) hStn1(WT) with hTen1(R27Q). (C) hStn1(WT) with hTen1(Y115A). (D) hStn1(WT) with hTen1(R119Q). (E) hStn1(D78A/l164A) with hTen1(WT). (F) hStn1(D78A/M167A) with hTen1(WT). (G) Table of ITC values for the full length, WT, single and double mutant hStn1 and hTen1 proteins obtained from the curve fit of figures $6 \mathrm{~A}-\mathrm{F}$.

doi:10.1371/journal.pone.0066756.g005

of a bound substrate and the weakest at the $3^{\prime}$ end, with RPA32 (hStnl homolog) on the far $3^{\prime}$ end [49]. Release of weakly associated ssDNA from hTenl may be the first step in sequential unbinding of the OB folds in the hCST complex to allow hand-off of a ssDNA substrate.

Another possibility is that the weak ssDNA binding activity of hTenl observed here is not biologically relevant and hTenl has a different role in hCST dependent telomere maintenance. The recently published structure of the 30 nucleotide binding mode of the RPA complex (RCSB ID: 4GOP) [49] provides a possible model for cooperative DNA binding between subunits of the hCST complex. The OB fold of RPA32 (hStnl homologue) is a DNA binding domain that is involved in the 30 nucleotide binding mode of RPA, and helps the complex to achieve its $0.1 \mathrm{nM}$ binding affinity for ssDNA while RPA14 (hTen1 homologue) is not involved in high-affinity DNA binding [49]. It's possible then that hTenl carries out primarily a structural role in hCST assembly thus enhancing Ctcl and hStnl [4] DNA binding via promoting the proper assembly of the trimeric CST complex. Without effective ssDNA binding hCST is not in position to cap telomeres effectively, leading to telomere elongation (Figure 6) and telomere damage (Figure 7) phenotypes observed in cells defective for hStnl-Tenl complex formation. hTenl may also act as a steric block, which prevents hStnl and possibly Ctcl from interacting with potential binding partners such as Pol $\alpha$. This notion is further supported by the lack of conserved solvent-exposed residues on
hTenl except for those involved in hStnl binding (Figure $4 \mathbf{B}$ and $\mathbf{C}$ ), Perhaps in the absence of hTenl, hStnl and Ctcl can interact with pol $\alpha$ to promote $\mathrm{C}$-strand synthesis.

\section{Materials and Methods}

\section{Protein Expression and Purification}

We designed the hStn $1 \mathrm{~N}$ construct using sequence alignment and secondary structure prediction with the PHYRE server [50]. The hStn1N gene consisting of residues 18-184, and carrying the his-MBP (Maltose Binding Protein) fusion tag at the N-terminus was overexpressed in Rosetta (DE3) pLysS cells (Millipore) using $1 \mathrm{mM}$ isopropyl- $\beta$-D-thiogalactopyranoside (IPTG; Gold Biotechnology) for 5 hours at $20^{\circ} \mathrm{C}$. hTen 1 with a N-terminal his tag was overexpressed in BL21-CodonPlus(DE3) RIPL cells (Stratagene) using $1 \mathrm{mM}$ IPTG for 3 hours at $20^{\circ} \mathrm{C}$. Cells were harvested by centrifugation and mixed together prior to lysis by sonication in $0.5 \mathrm{M} \mathrm{KCl}, 25 \mathrm{mM}$ Tris, $15 \mathrm{mM}$ imidazole, $5 \%$ glycerol, $0.1 \mathrm{mM}$ phenylmethylsulfonyl fluoride (PMSF), $0.1 \mathrm{mM}$ benzamidine, pH 7.5 (buffer A). The hStn1-Tenl purified using Superflow NiNitrilotriacetic acid (Ni.NTA; Qiagen) column, followed by an amylose resin (NEB) column. The fusion tags were cleaved by TEV overnight at $4^{\circ} \mathrm{C}$. Tandem Poros-HS and HQ columns (Perspective Biosystems) were used to remove residual contaminants and a Superdex S75 (GE Healthcare) to remove any aggregates. The full-length hStnl was overexpressed and purified 
A

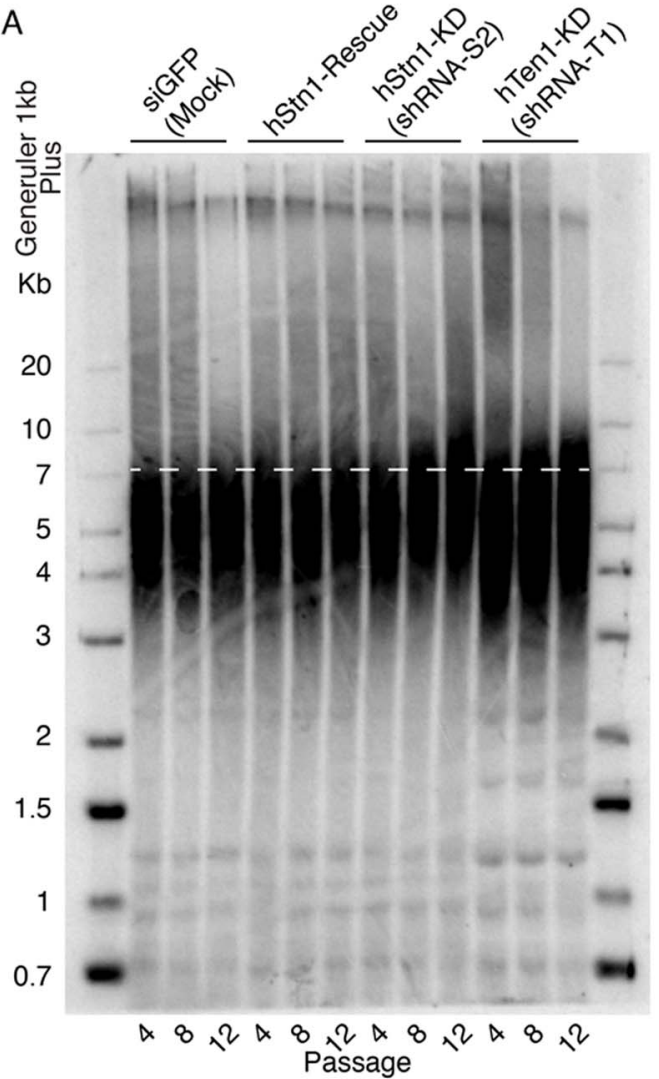

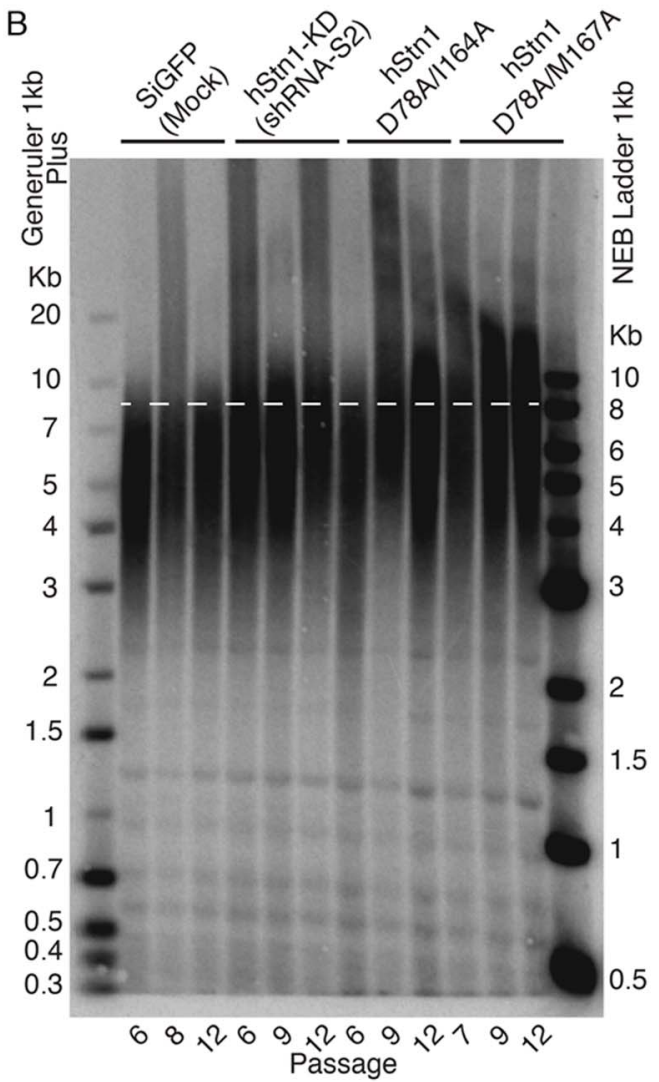

Figure 6. Southern blots analysis of telomeric DNA from cells carrying wild type (WT) or mutant hStn 1 defective of hTen 1 binding. (A) The gel shows telomere length at passages 4, 8 and 12 of cells carrying siGFP (Mock), hStn1-Rescue with WT protein, hStn1-KD (shRNA-S2) and hTen1-KD (shRNA-T1). The hStn1-KD (shRNA-S2) and double mutants defective of hTen1 binding show increased telomere length, compared to the siGFP (Mock). (B) The gel shows telomere length at passages 6, 7-9 and 12 of cells carrying siGFP (Mock), hStn1-KD (shRNA-S2) and the double mutants hStn1(D78A//164A) and hStn1(D78A/M167A).

doi:10.1371/journal.pone.0066756.g006

using the same procedure as that used for the hStnlN-Tenl complex.

\section{Protein Crystallization and Data Collection}

hStn1N-Ten1 crystallization. We concentrated the purified hStn $1 \mathrm{~N}-T e n 1$ complex to $18 \mathrm{mg} / \mathrm{mL}$ and dialyzed it for 3 hours in a buffer containing $100 \mathrm{mM} \mathrm{KCl}, 5 \mathrm{mM}$ Tris, $1 \mathrm{mM}$ TCEP, pH 7.5. hStnlN-Tenl crystals of the orthorhombic space group $\mathrm{P} 2{ }_{1} 2{ }_{1} 2$ grew under the sitting drop vapor diffusion method at room temperature in one week from $1 \mu \mathrm{L}$ drops containing the protein complex and $0.8 \mathrm{M} \mathrm{AmSO}_{4}, 0.1 \mathrm{M}$ citric acid $\mathrm{pH} 4.0$ and $5 \%$ jeffamine M-600. Streak seeding into drops prepared by mixing $1 \mu \mathrm{L}$ of hStn $1 \mathrm{~N}-\mathrm{Ten} 1$ with $1 \mu \mathrm{L}$ of $0.6 \mathrm{M} \mathrm{AmSO} 4,0.2 \mathrm{M}$ Citric Acid pH 4.0, 5\% jeffamine M-600, optimally reproduced the crystals. Crystals were harvested using a cryoprotectant consisting of $0.6 \mathrm{M} \mathrm{AmSO}$, $0.2 \mathrm{M}$ Citric Acid pH 4.0, 5\% jeffamine M-600, 30\% ethylene glycol. Data were collected at the National Synchrotron Light Source (NSLS) X25 beamline and processed using HKL2000 (Table 1 and 2). The crystals contained two hStn1-Tenl dimers in the asymmetric unit.

hStn1G crystallization. We concentrated the purified hStnlC to $41 \mathrm{mg} / \mathrm{mL}$ and dialyzed it for 3 hours in a buffer containing $100 \mathrm{mM} \mathrm{KCl,} 5 \mathrm{mM}$ Tris-HCl, $1 \mathrm{mM}$ TCEP, pH 7.5. hStnlC crystals of the $\mathrm{P} 2{ }_{1} 2_{1} 2_{1}$ space group grew under the microbatch method at room temperature in two weeks from $1 \mu \mathrm{L}$ drops containing hStnlC and $1.6 \mathrm{M}$ ammonium phosphate dibasic, $100 \mathrm{mM}$ Tris $\mathrm{pH} 8.5$ and 3\% ethylene glycol. Crystals were harvested using a cryoprotectant consisting of $1.6 \mathrm{M}$ ammonium phosphate dibasic, $100 \mathrm{mM}$ Tris-HCl, $\mathrm{pH} 8.5,3 \%$ ethylene glycol and 30\% glycerol. Data were collected at the National Synchrotron Light Source (NSLS) X25 beamline and processed using HKL2000 (Table 1 and 2). The crystals contained one hStnlC molecule in the asymmetric unit.

\section{Structure Determination and Refinement}

We used the method of multi-wavelength anomalous dispersion (MAD) experiment and mercury derivatized crystals to obtain initial phases. Mercury derivatives of hStnlN-Tenl and hStnlC were prepared by incubating the crystals with $5 \mathrm{mM}$ of $\mathrm{MeHgCl}$ for 15 minutes. Mercury sites for hStn $1 \mathrm{~N}-\mathrm{Ten} 1$ and $\mathrm{hStn} 1 \mathrm{C}$ were found using SOLVE [51] (Table 1 and 2), and refined in MLPHARE [52] with phase extension to $2.05 \AA$ and $1.6 \AA$ resolution using a native dataset. Density modification and model building was done in RESOLVE [53,54] with two-fold noncrystallographic symmetry (NCS) for hStnl-Tenl. The final model was built in COOT [55] and refined using REFMAC [56] (Table 1 and 2). The atomic coordinates and structure factors have been deposited in the Protein Data Bank (RCSB - www.pdb. org) under the accession numbers $4 \mathrm{JOI}$ and $4 \mathrm{JQF}$. 


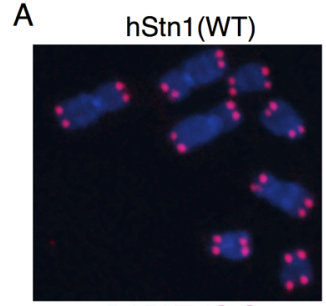

B

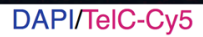

hStn1-KD/(shRNA-2)

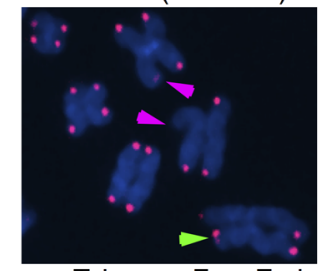

- Telomere Free Ends

C hStn1(D78A/l164A)

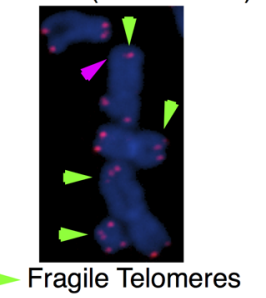

D

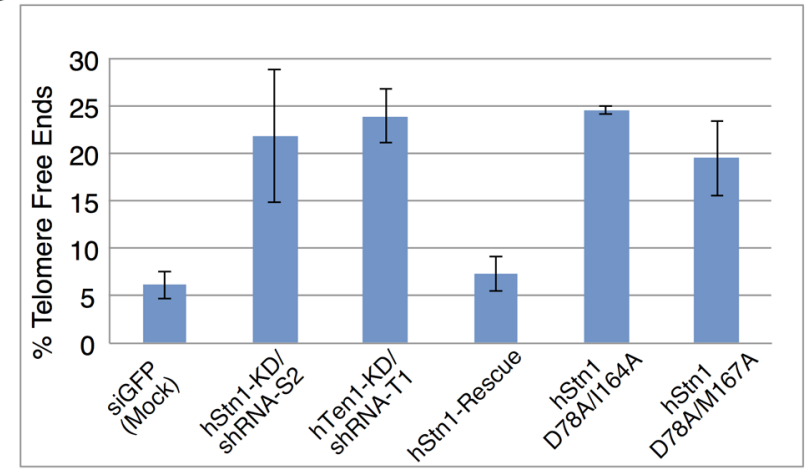

$\mathrm{E}$

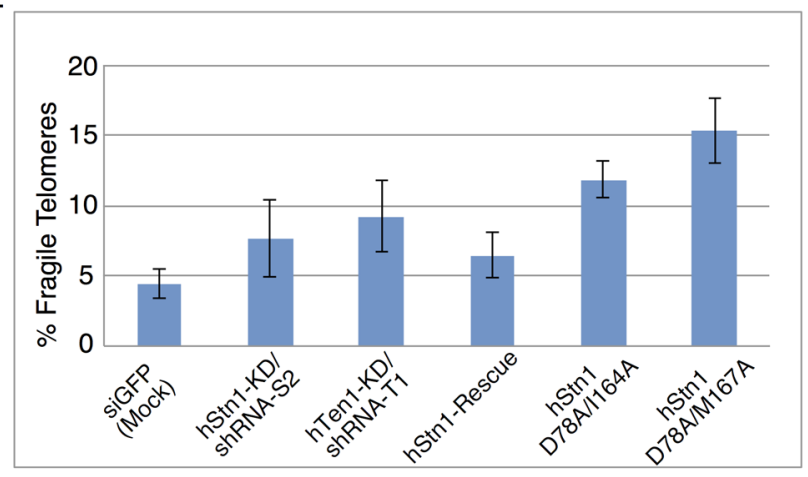

Figure 7. Fluorescence in situ hybridization (FISH) data of HEK 293 cells infected with: siGFP (Mock), hStn1-KD (shRNA-S2), hTen1KD (shRNA-T1), hStn1-Rescue and hStn 1 double mutants, hStn1(D78A/I164A) and hStn1(D78A/M167A),defective of hTen1 binding. (A) FISH of chromosomes in mock-treated (siGFP) cells display normal telomeres. (B) and (C) Telomere defects observed in hStn1 knockdown (hStn1-KD/shRNA-S2) and the double mutant hStn1(D78A/I164A) that disrupts hStn1-Ten1 association. Green arrows point to fragile telomeres, and pink to telomere free ends. (D) Bar graph showing the levels of telomere free ends observed in siGFP(mock), hStn1-Rescue and hStn1(D78A/I164A) or hStn1(D78a/M167A) double mutants defective of hTen1 binding. (E) Bar graph showing the levels of fragile telomeres observed in siGFP(mock), hStn1-Rescue and hStn1(D78A/I164A) or hStn1(D78a/M167A) double mutants and mutant hStn1 defective of hTen1 binding. Error bars show the standard deviation from 3 independent experiments. An average of $\sim 1000$ chromosomes were counted in each experiment.

doi:10.1371/journal.pone.0066756.g007

\section{Isothermal Titration Calorimetry (ITC)}

We carried out ITC experiments on a MicroCal iTC200 (GE). Purified full-length hStnl and hTenl (WT and mutants) were buffer exchanged into $(300 \mathrm{mM} \mathrm{KCl}, 25 \mathrm{mM}$ Tris-HCl, pH 7.5, $1 \mathrm{mM}$ DTT, 5\% glycerol). hTen 1 was injected at a concentration of $125 \mu \mathrm{M}$ into the cell containing hStnl at $12 \mu \mathrm{M}$. For the ITC runs, the cell of the calorimeter was kept at $20^{\circ} \mathrm{C}$, and the volume of each injection was $2.47 \mu \mathrm{L}$. Analysis of ITC data used the Origin analysis software (GE Healthcare) to obtain binding constants and ratios.

\section{Fluorescence Polarization (FP)}

15 binding reactions were prepared with $1 \mathrm{mg} / \mathrm{mL}$ BSA $100 \mathrm{mM} \mathrm{NaCl}, 5 \mathrm{mM} \mathrm{MgCl}$, $1 \mathrm{mM}$ DTT, 5\% glycerol, $2.5 \mathrm{nM}$ 6-FAM labeled DNA oligo, and various concentrations of purified full length hStn1, hTen1, or hStn1-Tenl complex. Reactions were mixed at room temperature in triplicate, transferred to an optiplate 384, and fluorescence polarization signal was measured using an Envision Xcite Multilabel Plate Reader (Perkin Elmer).

\section{Human Cell Culture}

Human cell culture studies were carried out in HEK 293T cells. The hStnl, hTenl genes in the pLU vector and shRNA in the pLKO.1 vector were delivered using lentiviral infection of the
HEK 293 T cells. We prepared lentiviral particles by lipofectomine $200{ }^{\circledR}$ (Invitrogen) transfection of HEK $293 \mathrm{~T}$ cells with the pLU or pLKO.1 and lentiviral production vectors. pLKO.1 vectors carrying the hStnl shRNAs and puromycin resistance were obtained from the Sigma Mission shRNA library (shRNA-S1, shRNA-S2, shRNA-S3, shRNA-S4 and shRNA-S5 correspond to TRCN 127870, 128123, 128703, 128801, and 129006 respectively). Four hTenl shRNAs were also tested from the Mission shRNA library (shRNA-T1, shRNA-T2, shRNA-T3, shRNA-T4 correspond to TRCN 337345, 447411, 337412, 371156) (Figure S6). The ectopic WT and mutant hStnl and hTenl genes used in this study were designed to carry silent mutations conferring resistance to the selected shRNAs. For protein expression we used pLU-EF1A-iBlast ( $\mathrm{pLU}$ ) vector, carrying blasticidin $\mathrm{S}$ resistance. Growth media was spiked with $5 \mu \mathrm{g} / \mathrm{mL}$ blasticidin $\mathrm{S}$, and $2 \mu \mathrm{g}$ / $\mathrm{mL}$ puromycin.

\section{Western Blot}

We tested the effectiveness of the Sigma Mission shRNAs against hStnl and hTenl in western blots using FLAG or hStnl and hTen 1 specific antibodies as well as RT-PCR. All three assays showed the same result. For the western blots, $3 \times 10^{6}$ HEK 293T cells were lysed in $300 \mu \mathrm{L}$ of pre-chilled RIPA buffer $(50 \mathrm{mM}$ Tris-HCl pH 7.5, $150 \mathrm{mM} \mathrm{NaCl,} 0.1 \%$ SDS, 0.5\% Sodium Deoxycholate, $1 \%$ Triton X-100). Lysates were centrifuged for 15 
minutes at $20,000 \times \mathrm{g}$ and the supernatant saved. Protein supernatants were quantified using a Bradford assay [57], and $20 \mu \mathrm{g}$ of each sample was run on a polyacrylamide gel. Protein blotted onto a polyvinylidene fluoride (PVDF) membrane (Perkin Elmer Health Sciences) by electrophoretic blot at $200 \mathrm{~mA}$ for 3 hours at $4^{\circ} \mathrm{C}$ in $20 \%$ methanol, $125 \mathrm{mM}$ Tris-HCl, $1.25 \mathrm{M}$ glycine and $0.5 \%$ SDS. The membrane was blocked with TBST (100 mM Tris-HCl, pH 7.5, 2 M NaCl, 0.5\% Tween-20) and 5\% bovine serum albumin (BSA), washed TBST, and then incubated overnight with monoclonal anti-FLAG antibody M2 produced in mouse (Sigma) in TBST at $4^{\circ} \mathrm{C}$. The membrane was further washed with TBST, incubated with the horseradish peroxidase (HRP)-conjugated anti-mouse IgG antibody (GE Healthcare) for 2 hours, and washed again with TBST. The chemiluminescent HRP reaction was activated using SuperSignal West Pico Substrate (Thermo Scientific), and the image developed with CL-exposure film (Thermo Scientific).

\section{Southern Blot Analysis of Telomere Length}

We extracted the genomic DNA from HEK 293T cells using the Qiagen QIAamp ${ }^{\circledR}$ DNA Mini Kit. We digested $10 \mu \mathrm{g}$ of genomic DNA with $1.5 \mu \mathrm{l}(15 \mathrm{U})$ of AluI (Invitrogen) and $3 \mu \mathrm{l}$ $(15 \mathrm{U})$ of $\mathrm{MboI}(\mathrm{NEB})$ endonucleases for 24 hours at $37^{\circ} \mathrm{C}$. Samples were ethanol precipitated overnight at $-20^{\circ} \mathrm{C}$ and pelleted by centrifugation, washed with $75 \%$ ethanol, and resuspended in $30 \mu \mathrm{l}$ of DNase free water. We loaded $3 \mu \mathrm{g}$ of each sample onto a $0.7 \% 1 \mathrm{X}$ Tris Acetate EDTA (TAE) agarose gel and run in $1 \times$ TAE buffer for 6 hours at $120 \mathrm{~V}$. The gel was then washed with $0.25 \mathrm{M} \mathrm{HCl}$, denaturing solution consisting of $0.5 \mathrm{M} \mathrm{NaOH}, 1.5 \mathrm{M} \mathrm{NaCl}$, followed by neutralization buffer $0.5 \mathrm{M}$ Tris-HCl, $3 \mathrm{M} \mathrm{NaCl}, \mathrm{pH}$ 7.5. After washing, the DNA was transferred from the gel to a hybridization transfer membrane (Genescreen Plus ${ }^{\text {TM }}$, Perkin Elmer Health Sciences) using capillary blotting. DNA was then UV cross-linked to the membrane using a UV Stratalinker 1800 (Stratagene), and hybridized with $0.2 \mathrm{nM}$ of ${ }^{32} \mathrm{P}$ labeled single stranded DNA probe (TTAGGG) $)_{4}$ overnight in $15 \mathrm{~mL}$ church buffer $7 \%$ SDS, $0.25 \mathrm{M} \mathrm{Na}_{2} \mathrm{PO}_{4} \mathrm{pH} 7.2,1 \mathrm{mM}$ EDTA, $1 \% \mathrm{w} / \mathrm{v}$ BSA). The nonhybridized probe was removed by washing with buffer containing $20 \mathrm{mM} \mathrm{Na}_{2} \mathrm{PO}_{4} \mathrm{pH} 7.2,1 \% \mathrm{w} / \mathrm{v}$ SDS and $1 \mathrm{mM}$ EDTA and the membrane was exposed to a phosphorimager overnight for sample imaging.

\section{Fluorescence in situ Hybridization (FISH)}

HEK 293T cells were grown to $70 \%$ confluence on a $10 \mathrm{~cm}$ plate and treated with $100 \mu \mathrm{g} / \mathrm{ml}$ of colcemid for $4 \mathrm{hrs}$. Cells were then trypsinized to detach them from the plate, pelleted and treated in a hypertonic environment $(75 \mathrm{mM} \mathrm{KCl}$ for 30 minutes at $37^{\circ} \mathrm{C}$ ) to rupture them. The cells were fixed in $10 \mathrm{ml}$ of $3: 1$ methanol:acetic acid solution and stored at $4^{\circ} \mathrm{C}$. Cells were dropped on frosted microscope slides (Thermo Scientific), rehydrated in coplin jars filled with phosphate buffered saline (PBS), fixed in 4\% formaldehyde (Sigma), permeabilized with $1 \mathrm{mg} / \mathrm{ml}$ pepsin in $10 \mathrm{mM}$ glycine, $\mathrm{pH} 2.0$ at $37^{\circ} \mathrm{C}$ (Sigma), and fixed again with $4 \%$ formaldehyde. Slides were then dehydrated in $70 \%, 95 \%$, then $100 \%$ ethanol successively, air dried, and hybridized with $20 \mu \mathrm{L}$ of $200 \mathrm{nM}$ telomeric-Cy5 peptide nucleic acid (PNA) probe (TelC-Cy5 - PNA biosciences) in 70\% formamide, $10 \mathrm{mM}$ Tris $\mathrm{pH}$ 7.5, 0.5\% Odyessy blocking buffer (LiCor) according to the manufacturer's instructions. Slides were stained with DAPI and imaged using a Nikon E600 upright microscope.

\section{Supporting Information}

Figure S1 Expression and purification data of the hStn1-Ten1 complex. Size exclusion chromatogram and SDS PAGE analysis of $(\mathbf{A})$ the full-length hStnl-Tenl complex. $(\mathbf{B})$ the hStn1N-Tenl complex $(\mathbf{G})$ the C-terminal domain of hStnl (hStnlG).

(TIF)

Figure S2 Structural homologs of hStn1C. (A) Overall structure of the G-terminal domain of hStnl with secondary structure elements labeled. (B) Structural alignment of hStn1C (pink) with $s c$ Stn $1 \mathrm{C}$ (green - PDB ID: $3 \mathrm{KEY}$ and $3 \mathrm{~K} 10)$. (G) and (D) Independent structural alignments of the two winged helix turn helix motifs of hStnl (wHTH1 and wHTH2) and $s c$ StnlC. (E) Alignment of the wHTH2 motif of hStnlC (pink) with that of RPA32 (blue - PDB ID: 1DPU).

(TIF)

Figure S3 Structural homologs of the hStn1-Ten1 complex. Structural alignment of hStn $1 \mathrm{~N}-\mathrm{Ten} l$ (wheat cartoon) with: (A) $s p$ Stn1N-Tenl (green - PDB ID: 3KF6) (B) $c t$ Stn1-Tenl (red PDB ID: 3KF8) and (G) RPA32-RPA14 (blue - PDB ID:1QUQ). (TIF)

Figure S4 Stn1, Ten1 and RPA sequence alignments. (A) hStnl sequence alignment with other Stn1 and RPA32; residues mutated in this study are shown in red color. (B) hTenl sequence alignment with other Tenl and RPA14; residues mutated in this study are shown in green color.

(TIF)

Figure S5 SDS-PAGE gel analysis of purified hStn1 and hTen1 wild type and mutant proteins used in ITC experiments. Lanes: 1) Markers 2) $\mathrm{hStnl}(\mathrm{WT}) \quad 3$ ) hStnl(D78A/I164A) 4) hStnl(D78A/M167A) 5) hTenl(WT) 6) hTen1(R27Q) 7) hTenl(Y115A) 8) hTen1(R119Q).

(TIF)

Figure S6 Western blots analysis of hStn1 and hTen1 expression in HEK 293T cellines. (A) Anti-flag tag Western blot showing the effect of five hStnl shRNAs on ectopic hStnl overexpression in the HEK 293 T cells. Lanes: 1) Markers 2) hStnl (WT) 3) shRNA-S1 4) shRNA-S2 5) shRNA-S3 6) shRNA-S4 and 7) shRNA-S5. (B) Anti-flag Western blot testing shRNA resistance of 3 different hStnl genes carrying silent mutations designed to prevent binding of shRNA-S1, 2 and 4. Lane 1) Markers; Lanes 27, HEK 293T cells co-infected with 2) shRNA-S1 resistant hStnl 3) siGFP and shRNA-S1 resistant hStnl 4) shRNA-S2 resistant hStnl 5) siGFP and shRNA-S2 resistant hStnl 6) shRNA-S4 resistant hStnl 7) siGFP and shRNA-S4 resistant hStnl (G) Antiflag tag Western blot showing the effect of anti-hTenl shRNAs on ectopic hTen l overexpression in the HEK 293T cells. Lanes: 1) Markers 2) untransfected HEK 293T cells 3) hTen1-pLU 4) shRNA-T1 5) shRNA-T2 6) shRNA-T3 and 7) shRNA-T4. (TIF)

\section{Acknowledgments}

We would like to thank Paul Lieberman and Zong Deng for reagents used in this project.

\section{Author Contributions}

Conceived and designed the experiments: ES. Performed the experiments: CB CR MH. Analyzed the data: ES CB CR MH. Contributed reagents/ materials/analysis tools: DS. Wrote the paper: ES CB. 


\section{References}

1. Blackburn EH, Gall JG (1978) A tandemly repeated sequence at the termini of the extrachromosomal ribosomal RNA genes in Tetrahymena. J Mol Biol 120: 33-53.

2. de Lange T (2009) How telomeres solve the end-protection problem. Science 326: 948-952.

3. Baumann P, Podell E, Cech TR (2002) Human Pot1 (protection of telomeres) protein: cytolocalization, gene structure, and alternative splicing. Molecular and cellular biology 22: 8079-8087.

4. Mivake Y, Nakamura M, Nabetani A, Shimamura S, Tamura M, et al. (2009) RPA-like mammalian Ctc1-Stn1-Ten1 complex binds to single-stranded DNA and protects telomeres independently of the Potl pathway. Molecular cell 36: 193-206.

5. Wellinger RJ (2009) The CST complex and telomere maintenance: the exception becomes the rule. Molecular cell 36: 168-169.

6. de Lange T (2005) Shelterin: the protein complex that shapes and safeguards human telomeres. Genes Dev 19: 2100-2110.

7. Pennock E, Buckley K, Lundblad V (2001) Cdc13 delivers separate complexes to the telomere for end protection and replication. Cell 104: 387-396.

8. Evans SK, Lundblad V (1999) Est1 and Cdc13 as Comediators of Telomerase Access. Science 286: 117-120.

9. Grandin N, Damon C, Charbonneau M (2001) Cdc13 prevents telomere uncapping and Rad50-dependent homologous recombination. The EMBO journal 20: 6127-6139

10. Puglisi A, Bianchi A, Lemmens L, Damay P, Shore D (2008) Distinct roles for yeast Stn1 in telomere capping and telomerase inhibition. The EMBO journal 27: 2328-2339.

11. Gao H, Cervantes RB, Mandell EK, Otero JH, Lundblad V (2007) RPA-like proteins mediate yeast telomere function. Nature structural \& molecular biology 14: $208-214$.

12. Qian W, Fu XH, Zhou JQ (2010) Purification and characterization of Stnlp, a single-stranded telomeric DNA binding protein. Protein expression and purification 73: 107-112.

13. Oian W, Wang J, Jin NN, Fu XH, Lin YC, et al. (2009) Tenlp promotes the telomeric DNA-binding activity of Cdc13p: implication for its function in telomere length regulation. Cell Res 19: 849-863.

14. Chandra A, Hughes TR, Nugent CI, Lundblad V (2001) Cdc13 both positively and negatively regulates telomere replication. Genes Dev 15: 404-414.

15. DeZwaan DC, Toogun OA, Echtenkamp FJ, Freeman BC (2009) The Hsp82 molecular chaperone promotes a switch between unextendable and extendable telomere states. Nature structural \& molecular biology 16: 711-716.

16. Li S, Makovets S, Matsuguchi T, Blethrow JD, Shokat KM, et al. (2009) Cdk1dependent phosphorylation of Cdc13 coordinates telomere elongation during cell-cycle progression. Cell 136: 50-61.

17. Tseng SF, Lin JJ, Teng SC (2006) The telomerase-recruitment domain of the telomere binding protein Cdc13 is regulated by Meclp/Tellp-dependent phosphorylation. Nucleic acids research 34: 6327-6336.

18. Petreaca RC, Chiu H-C, Eckelhoefer HA, Chuang C, Xu L, et al. (2006) Chromosome end protection plasticity revealed by Stn $1 \mathrm{p}$ and Ten $1 \mathrm{p}$ bypass of Cdc13p. Nat Cell Biol 8: 748-755.

19. Garvik B, Carson M, Hartwell L (1995) Single-stranded DNA arising at telomeres in cdc13 mutants may constitute a specific signal for the RAD9 checkpoint. Molecular cell 15: 6128-6138.

20. Bianchi A, Negrini S, Shore D (2004) Delivery of Yeast Telomerase to a DNA Break Depends on the Recruitment Functions of Cdc13 and Est1. Molecular cell 16: 139-146.

21. Lydall D, Weinert T (1995) Yeast checkpoint genes in DNA damage processing: implications for repair and arrest. Science 270: 1488-1491.

22. Lin JJ, Zakian VA (1996) The Saccharomyces CDC13 protein is a single-strand TG1-3 telomeric DNA-binding protein in vitro that affects telomere behavior in vivo. Proceedings of the National Academy of Sciences of the United States of America 93: 13760-13765.

23. Nugent CI, Hughes TR, Lue NF, Lundblad V (1996) Cdc13p: a single-strand telomeric DNA-binding protein with a dual role in yeast telomere maintenance. Science 274: 249-252.

24. Grandin N, Damon C, Charbonneau M (2001) Tenl functions in telomere end protection and length regulation in association with Stn1 and Cdc13. The EMBO journal 20: 1173-1183.

25. Martin V, Du LL, Rozenzhak S, Russell P (2007) Protection of telomeres by a conserved Stn1-Tenl complex. Proceedings of the National Academy of Sciences of the United States of America 104: 14038-14043.

26. Surovtseva YV, Churikov D, Boltz KA, Song X, Lamb JC, et al. (2009) Conserved telomere maintenance component 1 interacts with STN1 and maintains chromosome ends in higher eukaryotes. Molecular cell 36: 207-218.

27. Gu P, Min JN, Wang Y, Huang C, Peng T, et al. (2012) CTC1 deletion results in defective telomere replication, leading to catastrophic telomere loss and stem cell exhaustion. The EMBO journal 31: 2309-2321.

28. Chen LY, Redon S, Lingner J (2012) The human CST complex is a terminator of telomerase activity. Nature 488(7412): 540544

29. Nakaoka H, Nishiyama A, Saito M, Ishikawa F (2012) Xenopus laevis Ctc1Stn1-Ten 1 (xCST) protein complex is involved in priming DNA synthesis on single-stranded DNA template in Xenopus egg extract. The Journal of biological chemistry 287: 619-627.

30. Casteel DE, Zhuang S, Zeng Y, Perrino FW, Boss GR, et al. (2009) A DNA polymerase-\{alpha\} \{middle dot $\}$ primase cofactor with homology to replication protein A-32 regulates DNA replication in mammalian cells. The Journal of biological chemistry 284: 5807-5818.

31. Huang C, Dai X, Chai W (2012) Human Stn1 protects telomere integrity by promoting efficient lagging-strand synthesis at telomeres and mediating C-strand fill-in. Cell Res 22(12): 1681-1695.

32. Song X, Leehy K, Warrington RT, Lamb JC, Surovtseva YV, et al. (2008) STN1 protects chromosome ends in Arabidopsis thaliana. Proceedings of the National Academy of Sciences of the United States of America 105: 1981519820.

33. Stewart JA, Wang F, Chaiken MF, Kasbek G, Chastain PD 2nd, et al. (2012) Human CST promotes telomere duplex replication and general replication restart after fork stalling. The EMBO journal 31: 3537-3549.

34. Sun J, Yu EY, Yang Y, Confer LA, Sun SH, et al. (2009) Stn1-Ten1 is an Rpa2Rpa3-like complex at telomeres. Genes Dev 23: 2900-2914.

35. Gelinas AD, Paschini M, Reyes FE, Heroux A, Batey RT, et al. (2009) Telomere capping proteins are structurally related to RPA with an additional telomerespecific domain. Proceedings of the National Academy of Sciences of the United States of America 106: 19298-19303.

36. Holm L, Rosenström P (2010) Dali server: conservation mapping in 3D. Nucl Acids Res 38: W545-549.

37. Bochkareva E, Korolev S, Lees-Miller SP, Bochkarev A (2002) Structure of the RPA trimerization core and its role in the multistep DNA-binding mechanism of RPA. The EMBO journal 21: 1855-1863.

38. Brush GS, Morrow DM, Hieter P, Kelly TJ (1996) The ATM homologue MEC1 is required for phosphorylation of replication protein $\mathrm{A}$ in yeast. Proceedings of the National Academy of Sciences of the United States of America 93: 15075-15080.

39. Dornreiter I, Erdile LF, Gilbert IU, von Winkler D, Kelly TJ, et al. (1992) Interaction of DNA polymerase alpha-primase with cellular replication protein A and SV40 T antigen. The EMBO journal 11: 769-776.

40. Kenny MK, Lee SH, Hurwitz J (1989) Multiple functions of human singlestranded-DNA binding protein in simian virus 40 DNA replication: singlestrand stabilization and stimulation of DNA polymerases alpha and delta. Proceedings of the National Academy of Sciences of the United States of America 86: 9757-9761.

41. Ivessa AS, Zhou JQ, Schulz VP, Monson EK, Zakian VA (2002) Saccharomyces Rrm3p, a 5' to 3' DNA helicase that promotes replication fork progression through telomeric and subtelomeric DNA. Genes Dev 16: 1383-1396.

42. Makovets S, Herskowitz I, Blackburn EH (2004) Anatomy and dynamics of DNA replication fork movement in yeast telomeric regions. Molecular and cellular biology 24: 4019-4031.

43. Miller KM, Rog O, Cooper JP (2006) Semi-conservative DNA replication through telomeres requires Tazl. Nature 440: 824-828.

44. Dart DA, Adams KE, Akerman I, Lakin ND (2004) Recruitment of the cell cycle checkpoint kinase ATR to chromatin during S-phase. The Journal of biological chemistry 279: 16433-16440.

45. Taggart AK, Teng SC, Zakian VA (2002) Estlp as a cell cycle-regulated activator of telomere-bound telomerase. Science 297: 1023-1026.

46. Wellinger RJ, Wolf AJ, Zakian VA (1993) Saccharomyces telomeres acquire single-strand TG1-3 tails late in S phase. Cell 72: 51-60.

47. Xu L, Petreaca RC, Gasparyan HJ, Vu S, Nugent CI (2009) TEN1 is essential for CDC13-mediated telomere capping. Genetics 183: 793-810.

48. Fanning E, Klimovich V, Nager AR (2006) A dynamic model for replication protein A (RPA) function in DNA processing pathways. Nucleic acids research 34: 4126-4137.

49. Fan J, Pavletich NP (2012) Structure and conformational change of a replication protein A heterotrimer bound to ssDNA. Genes \& development 26: 2337-2347.

50. Kelley LA, Sternberg MJ (2009) Protein structure prediction on the Web: a case study using the Phyre server. Nat Protoc 4: 363-371.

51. Terwilliger TC, Berendzen J (1999) Automated MAD and MIR structure solution. Acta Crystallogr D Biol Crystallogr 55: 849-861.

52. Otwinowski Z (1991) Isomorphous Scattering and Anomalous Replacement. Warrington: Daresbury Laboratory.

53. Terwilliger $\mathrm{T}$ (2003) Automated main-chain model building by template matching and iterative fragment extension. Acta Crystallographica Section D 59: 38-44.

54. Terwilliger TC (2000) Maximum-likelihood density modification. Acta Crystallogr D Biol Crystallogr 56: 965-972.

55. Emsley P, Cowtan KD (2004) Acta Cryst D60. Acta Crystallogr D Biol Crystallogr: 2126-2132.

56. Murshudov GN, Vagin AA, Dodson EJ (1997) Refinement of Macromolecular Structures by the Maximum-Likelihood Method. Acta Crystallographica Section D 53: 240-255.

57. Bradford MM (1976) A rapid and sensitive method for the quantitation of microgram quantities of protein utilizing the principle of protein-dye binding. Analytical Biochemistry 72: 248-254. 